\title{
REFLEXIONES Y RECOMENDACIONES SOBRE EDUCACIÓN EN INFORMÁTICA
}

Carlos Zozaya*

\section{Introducción}

La importancia de la ciencia y la tecnología en el desarrollo de un país ha sido señalada en repetidas ocasiones por diversos autores. Salam ${ }^{1}$ afirma que el nivel de vida de una nación depende en gran medida de la generación y el uso de la ciencia y la tecnología. Por su parte, Toffler ${ }^{2}$ menciona al conocimiento como uno de los tres elementos sustentantes del poder, y califica la aparición de las tecnologías de información como el cambio más importante dentro de lo que él denomina la "revolución del conocimiento". Asimismo, De Alba ${ }^{3}$ y Gamboa ${ }^{4}$ comentan el papel que juega la educación como un elemento primordial en la creación de una infraestructura que permita a nuestro país la generación y no solamente el uso de la tecnología, mediante un fortalecimiento de la inves-

* Instituto Tecnológico Autónomo de México.

${ }^{1}$ Salam, A., "Ciencia y desarrollo", Rev. Ciencia y Desarrollo, número 75, 1987, México, Conacyt.

${ }^{2}$ Toffler, A., El cambio del poder: Conocimientos, bienestar y violencia en el umbral del siglo XXI, 1990, Plaza \& Janés.

${ }^{3}$ De Alba, E., "El futuro de la investigación", en México al filo del año 2000, 1989, ITAM.

${ }^{4}$ Gamboa, R., "Necesidades de una educación en aspectos técnicos", en México al filo del año 2000, op. cit. 
Carlos Zozaya

tigación en esta área y una incorporación temprana del quehacer científico en los currícula.

Por otra parte, la evolución de las tecnologías de información y su incorporación existente o potencial a un buen número de actividades, procesos, productos y servicios, hacen fundamental reflexionar sobre qué tipo de educación requieren los profesionistas que generarán y harán uso de esta tecnología en el futuro.

Esta reflexión no es fácil de realizar. Por un lado, la vertiginosa evolución de las tecnologías de información hacen difícil pensar en modelos curriculares ortodoxos que tienen el riesgo de volverse obsoletos en un corto plazo. Por otro, la necesidad de formar profesionistas que tengan una visión amplia del mundo, con base en una formación humanista y universal, impone retos a todos los agentes e insumos que contribuyen en el proceso educativo.

Este artículo es el producto de algunas reflexiones sobre diversos aspectos relacionados con la educación en informática en nuestro país. El texto comienza por caracterizar a la informática con una disciplina propia que resulta de una convergencia de diferentes áreas del conocimiento, y por presentar algunas de las razones por las cuales su estudio debe considerarse como una actividad fundamental en nuestro proceso

130 educativo. A continuación, se describe un análisis sobre la situación de la educación y de la investigación en informática en México con base en información proporcionada por el INEGI. ${ }^{5}$ Posteriormente, se discuten algunos lineamientos que podrían incorporarse en la definición de estrategias y políticas educativas con miras a impulsar a la informática en nuestro país.

\section{Naturaleza e importancia de la informática}

La informática debe concebirse en un sentido amplio y con un carácter propio. Si bien no existe una definición precisa del alcance de esta disciplina, es importante señalar que la misma ha surgido como una convergencia durante varias décadas entre las telecomunicaciones, las

${ }^{5}$ Situación de la informática en México, Edición 1994, Instituto Nacional de Estadística Geografía e Informática. 


\section{EDUCACIÓN EN INFORMÁTICA}

ciencias de la computación y la microelectrónica, incorporando a su vez conceptos y técnicas de la ingeniería, la administración, la psicología y la filosofía, entre otras disciplinas. Algunas áreas de la informática como es la de la inteligencia artificial tienen una estrecha relación con los algoritmos de búsqueda y de optimización de la investigación de operaciones y con los conceptos de psicología cognitiva.

Por otro lado, es importante notar que la informática tiende a trivializarse como resultado de la accesibilidad al uso de las tecnologías de información. Esta trivialización se manifiesta con actitudes simplistas que buscan reducir el alcance de la educación en informática a cursos de capacitación sobre el uso de las tecnologías, o a actitudes derrotistas que descartan cualquier posibilidad de que nuestro país participe en el proceso global de investigación e innovación en este tipo de tecnologías.

Desde un punto de vista puramente pragmático, la informática es importante por tres razones principales. ${ }^{6}$ En primer término, la informática ha demostrado que puede dar valor agregado a los bienes y servicios de una organización, porque permite transformarlos o mejorar la coordinación de las actividades relacionadas con el proceso de generación de éstos. ${ }^{7}$ Asimismo, la informática puede ayudar a transformar la manera en que una organización compite, afectando las fuerzas que controlan la competencia en una industria. Gracias a la informática, algunas organizaciones han podido crear barreras de entrada, reducir la amenaza de productos o servicios sustitutos, cambiar su forma de competir de costos a diferenciación o a especialización, y aumentar su poder de proveedores o de compradores. ${ }^{8}$ Finalmente, y posiblemente ésta sea la razón más importante, la informática puede ayudar a reinventar la manera como una organización opera. La mayoría de los procesos de una organización operan de acuerdo a reglas obsoletas y no toman en cuenta las ventajas que proporcionan las tecnologías de información. Las bases de datos compartidas que permiten tener información accesible en

${ }^{6}$ Zozaya, C., "Oportunidades para el uso de las tecnologías de información en las organizaciones", Rev. Soluciones Avanzadas.

${ }^{7}$ Porter, M. y Millar, V. E., "How Information gives you Competitive Advantage", en Harvard Business Review, Julio-Agosto 1985, p. 149-60.

${ }^{8}$ McFarlan, F. W., "Information Technology changes the way you compete", en Harvard Business Review, Mayo-Junio 1984, p. 98-103. 
Carlos Zozaya

diferentes puntos en forma simultánea, el uso de los sistemas expertos para representar y utilizar el conocimiento, y el uso de redes para intercambiar información, son solamente algunas de las nuevas tecnologías que nos permiten rediseñar la manera como operan las organizaciones.

A las razones anteriormente expuestas, habría que agregar muchas otras relacionadas con el potencial que brindan estas tecnologías para modilicar prácticamente todas nuestras actividades, incluyendo las relacionadas con el proceso educativo mismo. Farah $^{9}$ señala algunas de las oportunidades y retos que brinda la teleinformática en el almacenamiento, acceso, presentación y manipulación de material didáctico e información, que constituye uno de los insumos fundamentales del proceso educativo. Por otro lado, el uso que han tenido las tecnologías de información para ahorrar energía y recursos naturales y para controlar la generación de desperdicios, resaltan la importancia de esta disciplina en la conservación de nuestro medio ambiente.

Adicionalmente, habría que comentar que la informática tiene una naturaleza horizontal y que, al igual que otras disciplinas como las matcmáticas, no está circunscrita a un sector especial de la industria o de los servicios. Los conceptos y paradigmas que en ella se estudian, tienen un carácter propio y constituyen una base de conocimientos y técnicas

132 que pueden ser aplicables a diferentes sectores de la economía.

\section{Educación e investigación en informática en México}

La matrícula de alumnos a nivel licenciatura en informática rebasa ya los 70,000 alumnos. Ello representa un $6.56 \%$ de la matrícula total de licenciaturas en el país y sitúa a los programas de informática en el cuarto lugar, en cuanto a matrícula se refiere, después de los programas de contaduría, derecho y administración. Por su parte, la matrícula de programas de formación de técnicos en informática ha tenido un crecimiento exponencial, de tal manera que en 1993 rebasaba ya los 170,000 alumnos.

${ }^{9}$ Farah, J.L., "Del libro a la teleinformática: una oportunidad para el desarrollo", en México al filo del año 2000, op. cit. 


\section{EDUCACIÓN EN INFORMÁTICA}

No obstante lo impactante que pudiesen resultar estos números, es importante señalar que la formación de recursos humanos especializados en informática es todavía incipiente. La gran mayoría de los programas existentes están orientados a formar usuarios de la informática y no a preparar profesionistas que puedan innovar en este campo. Sin emitir un juicio severo a las instituciones educativas que imparten este tipo de programas, el Grupo Consultivo de Informática recientemente creado por el INEGI considera que existen menos de 10 instituciones con la posibilidad de desarrollar programas a fondo de especialización de recursos humanos. ${ }^{10}$ Esto es consecuencia de las necesidades de profesorado y material de instrucción necesario, las cuales crecen día a día debido a la vertiginosa evolución que tienen las tecnologías de información.

Lo anterior queda de manifiesto al revisar la relación que guarda la investigación en informática con los programas de posgrado en esta disciplina. Si bien la matrícula ha crecido a poco más de 1,500 alumnos, solamente representan un $2 \%$ de la matrícula de licenciatura. Asimismo, el número de investigadores en este campo es muy pequeño, llegando solamente a 270 . Por otro lado, los niveles salariales en la industria y en los negocios son significativamente superiores a los de las universidades y centros de investigación, por lo cual muchos de ellos abandonan sus actividades académicas.

Los resultados de una encuesta sobre proyectos de investigación en informática aplicada por el INEGI a 38 centros de investigación entre los cuales se incluyeron universidades, empresas, y laboratorios de investigación, muestran que en nuestro país solamente se están desarrollando 57 proyectos de investigación en informática, de los cuales apenas un $17.5 \%$ se refiere a investigación básica. Además, únicamente tres de estos proyectos tienen un presupuesto superior a los 200,000 dólares, lo cual demuestra la visión a corto plazo que se tiene de impulsar la investigación en este campo.

Adicionalmente, habría que comentar que no existen programas académicos que vinculen estrechamente a la informática con las demás disciplinas en un mismo plan de estudios. La carencia de este tipo de

${ }^{10}$ Elementos para un plan estratégico en informática, Grupo Consultivo en Política Informática, Instituto Nacional de Estadística Geografía e Informática (en elaboración). 
Carlos Zozaya

programas refuerza la desafortunada concepción del informático como un tecnólogo de bata blanca que solamente conoce de las tecnologías per $s e$, pero que no cuenta con conocimientos sobre la aplicabilidad de estas tecnologías en las demás disciplinas. Si estamos de acuerdo en que la informática puede ayudar a reinventar la manera como una organización opera, es importante crear nuevos programas híbridos que den a los profesionistas una visión no solamente tecnológica sino más universal.

\section{Reflexiones y recomendaciones}

Habiendo comentado el papel de la informática como una disciplina fundamental en el desarrollo científico y tecnológico de una sociedad, y presentado un resumen de la situación de la educación y de la investigación en informática en nuestro país, es necesario proceder a hacer algunas reflexiones y recomendaciones con relación al proceso educativo.

En un sentido amplio, la educación no debe concebirse como un mecanismo de adquisición de conocimientos sino como un proceso, en permanente perfeccionamiento, que afecta a todas las facetas de la personalidad global de un individuo. Los nuevos modelos educativos del siglo $\mathrm{XX}$ han propuesto una visión más enriquecedora de este proceso. Las teorías de Decroly y de Freinet sobre una educación activa basada en el trabajo en equipo, así como las de Neill y sus discípulos sobre la educación anti-autoritaria demostraron la importancia de concebir al educando como un individuo con personalidad, intereses, contexto y cualidades propias.

George Williams ${ }^{11}$ subraya el daño que causan aquellos profesores que no pueden hacer nada más que ridiculizar a sus estudiantes, que son víctimas de una óptica puramente ligada a los hechos y que inhibe cualquier indicio de imaginación o creatividad, que son tan apegados a la "verdad". que desaniman cualquier pensamiento libre, o que son tan autoritarios e inflexibles que quitan a los alumnos toda la posibilidad de disfrutar de la clase.

${ }^{11}$ Williams, G., Some of my Best Friends are Professors, 1958, Abelard-Schuman L. 


\section{EDUCACIÓN EN INFORMÁTICA}

En esta misma línea, De la Isla ${ }^{12}$ subraya la importancia de promover el cultivo de la imaginación, de la reflexión y de la inventiva, en contraposición al sistema tradicional de comprensión, conservación y repetición de los conocimientos programados en el proceso educativo. Sugiere mantener una mente abierta que permita explorar lo desconocido, y propone una nueva metodología pedagógica con base en seminarios abiertos dirigidos a despertar este tipo de actividades.

Esta inquietud es compartida por otros autores. En un estudio sobre la manera en la que los seres humanos resuelven problemas, Hayes ${ }^{13}$ resalta el papel de la creatividad y de la invención como aptitudes fundamentales para todas las actividades del ser humano y señala que gran parte de lo que se conoce como "inteligencia" depende de la adquisición de conocimientos especializados, los cuales deben tener un nivel de abstracción tal, que los haga útiles para diferentes dominios de aplicación. En particular, es importante que los estudiantes adquieran conocimientos especializados sobre cómo representar los problemas, sobre cómo inventar alternativas de solución, y sobre cómo seleccionar entre estas alternativas para tomar una buena decisión.

Sin embargo, la adquisición de conocimientos especializados y el estímulo de aptitudes creativas e innovadoras debe ser complementado con metodologías pedagógicas para que los estudiantes aprendan a aprender. En este sentido, es imperativo evitar actitudes impositivas o paternalistas en el proceso educativo. La asistencia al aula es solamente uno de los múltiples mecanismos que contribuyen a la formación de una persona; los estudiantes como miembros de una sociedad reciben muchos otros insumos de información que afectan su formación. Por ello, es importante que los planes de estudio contengan cursos de humanidades que amplíen y cuestionen su visión del mundo y que les den elementos para discriminar los insumos de información que reciben en su contexto social.

${ }^{12}$ De la Isla, C., "De la educación de la memoria al desarrollo de la imaginación", en México al filo del año 2000, op. cit.

${ }^{13}$ Hayes, J. R., The Complete Problem Solver, 1989, Lawrence Erlbaum Associates Publishers. 
Carlos Zozaya

En una descripción de la educación transpersonal, Ferguson ${ }^{14}$ propone que el proceso educativo sea bidireccional en lugar de unidireccional, para que los alumnos tengan una participación más activa; que ponga más énfasis en elementos que permitan a los alumnos aprender a aprender, que en el contenido del material de estudio; y que busque estimular ambos lados del cerebro y no sólo el hemisferio izquièrdo, asociado con los procesos intelectuales.

La opinión anterior es compartida por Lyon, ${ }^{15}$ quien hace una crítica del sistema educativo tradicional que solamente busca desarrollar las habilidades intelectuales de los estudiantes y que deja a un lado la parte afectiva de la persona. Lyon señala que la educación integral debe considerar a los sentimientos como una parte primordial de la naturaleza humana y, por lo tanto, del proceso educativo. Es necesario aprender a sentir, y sentir para aprender.

Las reflexiones anteriores nos llevan a una concepción más integral del proceso educativo, la cual me parece fundamental. Un profesionista sin imaginación e inventiva, sin conocimientos adecuados o sin amor por lo que hace, no es un profesionista completo. El licenciado o ingeniero en informática debe ser creativo para encontrar nuevas soluciones, para identificar oportunidades de mejoramiento y para aprovechar sus conocimientos en el uso de las tecnologías de información. En este sentido, la actualización permanente de los planes de estudio, la incorporación de laboratorios que permitan a los estudiantes jugar con tecnología de punta y que estimulen la invención de nuevos dispositivos o aplicaciones, así como la participación de alumnos en actividades de investigación junto con los profesores, son algunos elementos que deben estar siempre presentes en la formación de profesionistas en esta área.

Por otro lado, la creatividad y la invención requieren ser estimuladas desde temprana edad. Es imposible pretender despertar estas aptitudes con solamente cuatro años de formación a nivel licenciatura. Como ya se mencionó, existe un buen número de investigadores trabajando en

${ }^{14}$ Ferguson, M., La conspiración de Acuario: Transformaciones personales y sociales en este fin de siglo, 1985, Madrid, Kairós.

${ }^{15}$ Lyon, H. C., Leaming to Feel - Feeling to Learn, 1971, Charles Merrill Publishing Co. 


\section{EDUCACIÓN EN INFORMÁTICA}

este campo, y habrá que estar abierto para analizar las alternativas pedagógicas que ellos propongan.

Con respecto al diseño de los currícula habría que reiterar la necesidad de contar con programas de estudio flexibles, que puedan mantenerse constantemente actualizados, así como adaptarse al contexto socioeconómico en el que serán aplicados. No creo que puedan definirse modelos curriculares rígidos o que exista un único conjunto de programas educativos que puedan ser aplicables en todo el país. En informática los programas educativos se vuelven obsoletos en muy corto tiempo, por lo que los cursos deben diseñarse de tal manera que puedan incorporar los avances conceptuales y tecnológicos en forma continua.

El contexto social que rodea a las instituciones de educación superior debe jugar un papel fundamental en el diseño de los currícula. En un artículo sobre política educativa para comunidades de bajos recursos económicos, Dávila ${ }^{16}$ señala que los hogares más pobres para el año 2,000 seguirán presentando características cualitativas similares a la que se han presentado en las últimas décadas. Considerando que una alta proporción de estos hogares se encuentran ubicados en zonas rurales y que sus miembros se ocuparán en labores agropecuarias. Sería conveniente que los programas de estudio dieran un mayor énfasis al uso de las tecnologías de información en este tipo de actividades.

Una política educativa completa no puede dejar a un lado a los adultos y considerar solamente a los jóvenes o niños en sus programas. La educación tiene un papel muy importante como medio de transformación social y de la realidad socioeconómica de los individuos. La demanda de carreras técnicas en informática demuestra el interés que existe por adquirir conocimientos que permitan tener un mejor trabajo. En este sentido, las experiencias y los conceptos de Paulo Freire en sus estudios sobre educación y alfabetización para adultos deben ser asimilados dentro de una política educativa. El uso de la palabra generadora de Freire como una manera de vincular al educando con su contexto propio sugiere la importancia de tomar en cuenta las experiencias adquiridas por el adulto en el diseño de una metodología pedagógica y de un currículum adecuado.

${ }^{16}$ Dávila C., E. R., "Los hogares más pobres en èl año 2000 y la política educativa", en México al filo del año 2000, op. cit. 


\section{Carlos Zozaya}

Por último, me gustaría comentar las oportunidades que dan las nuevas tecnologías de información para modificar al proceso educativo en sí. Los nuevos medios de comunicación son más poderosos, accesibles, interactivos y fáciles de usar que sus predecesores. Asimismo, la miniaturización y el bajo costo de las tecnologías de información abren la oportunidad de desarrollar aplicaciones de esta tecnología para apoyar el proceso educativo. Desafortunadamente, como lo menciona Toffler, ${ }^{17}$ falta mucho por investigar con relación a los vínculos entre la educación y los nuevos medios de comunicación. Si bien se tiene un riesgo de contar con un universo de información que produzca el overflow que menciona Farah, ${ }^{18}$ seguramente los avances de la inteligencia artificial producirán nuevas herramientas que permitan jerarquizar, interpretar, filtrar y aprovechar esta información de manera adecuada.

En resumen, y con base en las reflexiones anteriormente comentadas, se pueden elaborar algunas recomendaciones sobre la educación de las tecnologías de información en nuestro país:

Es necesario concebir a la educación en general, y a la educación en informática en particular, con un enfoque amplio e integral que considere todos los aspectos relacionados con la persona y su contexto.

El currículum de los programas de estudio en informática debe ser flexible, dinámico y adecuado al contexto social donde va a ser aplicado. Asimismo, se deben considerar las características propias del educando dentro de la planeación de las metodologías y contenidos, lo cual es particularmente importante en los programas de educación para adultos.

El proceso educativo debe de estimular la creación y la imaginación, así como la comprensión de conocimientos especializados que permitan a los alumnos adquirir las aptitudes necesarias para enfrentarse a la solución de problemas y a la toma de decisiones.

${ }_{17}^{17}$ Toffler, A., op. cit.

${ }^{18}$ Farah, J.L., op. cit. 


\section{EDUCACIÓN EN INFORMÁTICA}

Es necesario impulsar nuevos programas híbridos, donde se combinen conocimientos de tecnologías de información con otras disciplinas, que den a los profesionistas informáticos una formación no solamente tecnológica sino más universal.

Asimismo, se requiere desarrollar nuevos programas de especialización que permitan crear una infraestructura suficientemente sólida para impulsar la investigación en esta área.

Finalmente, es importante continuar la investigación sobre la manera como la informática va a modificar al proceso educativo.

Desde luego, estas reflexiones y recomendaciones no pretenden ser exhaustivas. En un tema tan apasionante como el de la educación, con seguridad hay múltiples aspectos que aprender y discutir. Manos, cerebros y corazones a la obra. 